Pacific

Journal of

Mathematics

THE MULTIPLICITY-ONE THEOREM FOR GENERIC AUTOMORPHIC FORMS OF GSp(4)

DIHUA JIANG AND DAVID SOUDRY

Volume $229 \quad$ No. 2

February 2007 


\title{
THE MULTIPLICITY-ONE THEOREM FOR GENERIC AUTOMORPHIC FORMS OF GSp(4)
}

\author{
DIHUA JIANG AND DAVID SOUDRY
}

\begin{abstract}
We prove the multiplicity-one theorem for irreducible, globally generic, cuspidal, automorphic representations of $\mathrm{GSp}_{4}(\mathbb{A})$ occurring in the space of cuspidal automorphic functions on $\mathrm{GSp}_{4}(\mathbb{A})$, where $\mathbb{A}$ is the ring of adeles of a totally real number field $k$.
\end{abstract}

In this note, we prove the multiplicity-one theorem for irreducible, globally generic, cuspidal, automorphic representations of $\mathrm{GSp}_{4}(\mathbb{A})$ occurring in the space of cuspidal automorphic functions on $\mathrm{GSp}_{4}(\mathbb{A})$, where $\mathbb{A}$ is the ring of adeles of a totally real number field $k$. This result has been expected for a long time. Recently, F. Shahidi encouraged us to write up a complete proof, which has an important application to the proof of the existence of certain stable Siegel modular forms of weight three on $\mathrm{GSp}_{4}$. See [Ramakrishnan and Shahidi 2007] for details.

The restriction that the number field $k$ is totally real stems from our use of [Kudla et al. 1992, Theorem 8.1], where this is an assumption on $k$. Otherwise, this restriction is not needed any further.

The proof we give is self-contained. Some of the ideas we use may be found in [Piatetski-Shapiro 1979]. To state the theorem, we denote by $L_{c}^{2}\left(\mathrm{GSp}_{4}, \xi\right)$ the space of cuspidal automorphic functions on $\mathrm{GSp}_{4}(\mathbb{A})$ with central character $\xi$.

Theorem. The multiplicity $m_{c}(\pi)$ of an irreducible, globally generic, cuspidal, automorphic representation $\pi$ occurring in the space of cuspidal automorphic forms, $L_{c}^{2}\left(\mathrm{GSp}_{4}, \xi\right)$, is one.

Proof. Let $B=T U$ be a Borel subgroup of $\mathrm{GSp}_{4}$, where $T$, the reductive part, is a maximal split torus and $U$, the unipotent radical, is a maximal unipotent subgroup. Then

$$
U /[U, U] \cong k \cdot X_{\alpha} \oplus k \cdot X_{\beta},
$$

where $\alpha$ is the short simple root and $\beta$ is the long simple root, and $X_{\alpha}$ (or $X_{\beta}$ ) is a basis vector in the one-dimensional $k$-root space of $\alpha$ (or $\beta$ respectively). Let $\psi$ be a character of $U(\mathbb{A})$ that is trivial when restricted to $U(k)$. Then $\psi$ factorizes

MSC2000: primary 11E70, 11E72; secondary 11E12.

Keywords: multiplicity-one theorem, automorphic forms.

Jiang is partially supported by NSF grant DMS-0400414. 
through $U(\mathbb{A}) / U(k)[U(\mathbb{A}), U(\mathbb{A})]$, which is isomorphic, as an abelian group, to $(\mathbb{A} / k) \cdot X_{\alpha} \oplus(\mathbb{A} / k) \cdot X_{\beta}$. We say that $\psi$ is generic if it is nontrivial on each of the simple root subgroups, via the isomorphism above. By Pontriagin duality, such characters of $U(\mathbb{A})$ are parametrized by pairs $\underline{a}=\left(a_{1}, a_{2}\right) \in\left(k^{\times}\right)^{2}$, once we fix a nontrivial character $\psi^{(0)}$ of $\mathbb{A} / k$. We denote the character corresponding to $\underline{a}$ by $\psi_{\underline{a}}^{(0)}$.

For any automorphic form $\phi$ on $\mathrm{GSp}_{4}(\mathbb{A})$ and generic character $\psi$ of $U(\mathbb{A})$, the $\psi$-Whittaker-Fourier coefficient of $\phi$ is defined by

$$
\mathcal{W}^{\psi}(\phi)(g)=\int_{U(k) \backslash U(\mathbb{A})} \phi(u g) \psi^{-1}(u) d u .
$$

If the integral on the right is nonzero as a function of $g$, we say that $\phi$ is $\psi$-generic. An automorphic representation $\pi$ of $\mathrm{GSp}_{4}(\mathbb{A})$, acting in a space of automorphic forms $V_{\pi}$, is called globally $\psi$-generic if there is some $\phi \in V_{\pi}$, which is $\psi$-generic. For $t \in T(k)$, since $\phi$ is automorphic, we have

$$
\begin{aligned}
\int_{U(k) \backslash U(\mathrm{~A})} \phi(u g) \psi^{-1}(u) d u & =\int_{U(k) \backslash U(\mathrm{~A})} \phi\left(t^{-1} u g\right) \psi^{-1}(u) d u \\
& =\int_{U(k) \backslash U(\mathbb{A})} \phi\left(u t^{-1} g\right) \psi^{-1}\left(t u t^{-1}\right) d u .
\end{aligned}
$$

If $\psi=\psi_{\underline{a}}^{(0)}$, we have

$$
\psi_{\underline{a}}^{(0)}\left(t u t^{-1}\right)=\psi_{t \cdot \underline{a}}^{(0)}(u),
$$

where

$$
t \cdot \underline{a}=\left(\alpha(t) \cdot a_{1}, \beta(t) \cdot a_{2}\right) .
$$

$T(k)$ acts transitively on the set of generic characters of $U(\mathbb{A})$. We conclude that any automorphic form $\phi$ on $\mathrm{GSp}_{4}(\mathbb{A})$, which is $\psi$-generic, is also generic with respect to each generic character of $U(\mathbb{A})$. This implies that, for an automorphic representation $\pi$ (acting in a space of automorphic forms) on $\mathrm{GSp}_{4}(\mathbb{A})$, the notion of global genericity is independent of the choice of generic characters of $U(\mathbb{A})$. In this case, we will say that $\pi$ is globally generic, once we know that it is globally generic with respect to some (and hence all) generic characters .

Let $F$ be a local field and let $\tau$ be a smooth representation of $\operatorname{GSp}_{4}(F)$, acting in a space $V_{\tau}$. As above, we have the notion of generic characters of $U(F)$, and they are parametrized by ordered pairs of elements in $F^{*}$, once we fix a nontrivial character of $F$. Given a generic character $\psi$ of $U(F)$, we say that $\tau$ is $\psi$-generic if there is a nontrivial linear functional $l$ on $V_{\tau}$ such that

$$
l(\pi(u) v)=\psi(u) l(v),
$$

for all $u \in U(F)$ and all $v \in V_{\tau}$. As above, if $\tau$ is $\psi$-generic, then it is $\psi^{\prime}$-generic, with respect to all generic characters $\psi^{\prime}$ of $U(F)$. 
Let $\pi$ be an irreducible automorphic representation of $\operatorname{GSp}_{4}(\mathbb{A})$, acting in a space of automorphic forms. We know that $\pi$ is isomorphic, as a representation, to a restricted tensor product $\pi \cong \otimes^{\prime} \pi_{v}$. We say that $\pi$ is locally generic at a place $v$ if $\pi_{v}$ is generic, and we say that $\pi$ is abstractly generic if $\pi$ is locally generic at all places. Clearly, if $\pi$ is globally generic, then $\pi$ is abstractly generic. The converse to this statement is not known.

For a given generic character $\psi$ of $U(\mathbb{A})$, define

$$
L_{0}^{2}\left(\mathrm{GSp}_{4}, \xi\right)=\left\{\phi \in L_{c}^{2}\left(\mathrm{GSp}_{4}, \xi\right) \mid \mathscr{W}^{\psi}(\phi) \equiv 0\right\} .
$$

It is clear that $L_{0}^{2}\left(\mathrm{GSp}_{4}, \xi\right)$ is a subspace of $L_{c}^{2}\left(\mathrm{GSp}_{4}, \xi\right)$. Let $L_{0^{\perp}}^{2}\left(\mathrm{GSp}_{4}, \xi\right)$ be the orthogonal complement of $L_{0}^{2}\left(\mathrm{GSp}_{4}, \xi\right)$ in $L_{c}^{2}\left(\mathrm{GSp}_{4}, \xi\right)$. Then

$$
L_{c}^{2}\left(\mathrm{GSp}_{4}, \xi\right)=L_{0}^{2}\left(\mathrm{GSp}_{4}, \xi\right) \perp L_{0^{\perp}}^{2}\left(\mathrm{GSp}_{4}, \xi\right),
$$

and for an irreducible cuspidal automorphic representation $\pi$, realized in a given subspace $V_{\pi} \subset L_{c}^{2}\left(\mathrm{GSp}_{4}, \xi\right)$, we have

$$
m_{c}(\pi)=m_{0}(\pi)+m_{0^{\perp}}(\pi),
$$

where $m_{0}(\pi)$ (or $m_{0^{\perp}}(\pi)$ ) is the multiplicity of $\pi$ occurring in $L_{0}^{2}\left(\mathrm{GSp}_{4}, \xi\right.$ ) (or in $L_{0^{\perp}}^{2}\left(\mathrm{GSp}_{4}, \xi\right)$, resp.). In order to prove the theorem, we show that

$$
m_{0}(\pi)=0 \quad \text { and } \quad \mathrm{m}_{0^{\perp}}(\pi)=1
$$

for all irreducible, globally generic, cuspidal, automorphic representations $\pi$ of $\mathrm{GSp}_{4}(\mathbb{A})$, with central character $\xi$.

We first note that

$$
m_{0^{\perp}}(\pi) \leq 1,
$$

for all irreducible, globally generic, cuspidal, automorphic representations $\pi$ of $\mathrm{GSp}_{4}(\mathbb{A})$, with central character $\xi$. The argument here is due to Piatetski-Shapiro [1979]. We need to show that any two isomorphic irreducible submodules $V_{\pi^{\prime}}, V_{\pi^{\prime \prime}}$ of $L_{0^{\perp}}^{2}\left(\mathrm{GSp}_{4}, \xi\right)$ affording the cuspidal representations $\pi^{\prime}$ and $\pi^{\prime \prime}$ are equal.

Let $\mathscr{T}: V_{\pi^{\prime}} \longrightarrow V_{\pi^{\prime \prime}}$ be an isomorphism as representations of $\mathrm{GSp}_{4}(\mathbb{A})$. Clearly $\pi^{\prime}$ and $\pi^{\prime \prime}$ are globally generic. By the local and global uniqueness of $\psi$-Whittaker models, there is a nonzero constant $c \in \mathbb{C}$, such that

$$
\mathcal{W}_{\mathcal{T}(\phi)}^{\psi}=c \cdot \mathcal{W}_{\phi}^{\psi} \text {. }
$$

It follows that

$$
\mathcal{W}_{\mathscr{T}(\phi)-c \cdot \phi}^{\psi}=\mathcal{W}_{\mathscr{T}(\phi)}^{\psi}-c \cdot \mathcal{W}_{\phi}^{\psi}=0 .
$$

Note that the space of all $\mathscr{T}(\phi)-c \phi$, as $\phi$ varies in $V_{\pi^{\prime}}$, is either zero or an irreducible submodule, isomorphic to $\pi^{\prime}$. We claim that $\mathscr{T}(\phi)-c \cdot \phi=0$, for all $\phi$ in 
$V_{\pi^{\prime}}$. Otherwise, if $\mathscr{T}(\phi)-c \cdot \phi \neq 0$ for some $\phi$, we get that the nonzero cuspidal automorphic form $\mathscr{T}(\phi)-c \cdot \phi$ generates an irreducible cuspidal automorphic subrepresentation $V_{\pi^{\prime \prime \prime}}$ in $L_{0}^{2}\left(\mathrm{GSp}_{4}, \xi\right)$. This is clear from (4) and (1). However, it is also clear that $V_{\pi^{\prime \prime \prime}}$ is contained in

$$
V_{\pi^{\prime}}+V_{\pi^{\prime \prime}} \subset L_{0^{\perp}}^{2}\left(\mathrm{GSp}_{4}, \xi\right),
$$

and this is a contradiction. This implies that $\mathscr{T}=c \cdot \mathrm{id}_{V_{\pi^{\prime}}}$, and therefore, $V_{\pi^{\prime}}=V_{\pi^{\prime \prime}}$.

Next, we show that $m_{0}(\pi)=0$ for all irreducible, globally generic, cuspidal, automorphic representations $\pi$ of $\mathrm{GSp}_{4}(\mathbb{A})$. Once we prove this, we get from (2) and (3) that

$$
1 \leq m_{c}(\pi)=m_{0^{\perp}}(\pi) \leq 1,
$$

and hence $m_{c}(\pi)=1$, as we want.

Assume that $m_{0}(\pi) \neq 0$. Then there is an irreducible subrepresentation $V_{\pi^{\prime}} \subset$ $L_{0}^{2}\left(\mathrm{GSp}_{4}, \xi\right)$ such that $\pi \cong \pi^{\prime}$, as representations of $\mathrm{GSp}_{4}(\mathbb{A})$. It follows that $\pi^{\prime}$ has the following two properties.

Property (a). $\pi^{\prime}$ is equivalent to an irreducible, globally generic, cuspidal, automorphic, representation $\pi$ of $\mathrm{GSp}_{4}(\mathbb{A})$ (in particular, $\pi^{\prime}$ is abstractly generic).

Property (b). $\pi^{\prime}$ has (identically) zero Whittaker-Fourier coefficients with respect to any generic character of $U(\mathbb{A})$.

We want to show that if such a $\pi^{\prime}$ exists for $\operatorname{GSp}_{4}(\mathbb{A})$, we can construct an irreducible cuspidal automorphic representation $\sigma^{\prime}$ of $\mathrm{Sp}_{4}(\mathbb{A})$ satisfying three properties about to be stated, where the notions of local genericity of a representation of $\mathrm{Sp}_{4}\left(k_{v}\right)$ and of global genericity of an automorphic representation of $\mathrm{Sp}_{4}(\mathbb{A})$ with respect to a given generic character are defined as in the case of $\mathrm{GSp}_{4}$.

Property 1. At each place $v$, the representation $\sigma_{v}^{\prime}$ is (locally) generic, with respect to some (local) generic character of $U\left(k_{v}\right)$. (We do not require that the local generic characters patch together to a global generic character of $U(\mathbb{A}) / U(k)$.)

Property 2. The representation $\sigma^{\prime}$ is nearly equivalent to an irreducible, cuspidal, automorphic representation $\sigma$ of $\operatorname{Sp}_{4}(\mathbb{A})$, which is globally generic, with respect to some generic character.

Property 3. The representation $\sigma^{\prime}$ has (identically) zero Whittaker-Fourier coefficients with respect to any generic character of $U(\mathbb{A})$.

However, by [Kudla et al. 1992, Theorem 8.1], there exists no irreducible cuspidal automorphic representation $\sigma^{\prime}$ of $\mathrm{Sp}_{4}(\mathbb{A})$ satisfying Properties 1, 2, and 3 for $\mathrm{Sp}_{4}(\mathbb{A})$. For this, we have to show that the degree 5 partial $L$-function of $\sigma^{\prime}$ does not vanish at $s=1$, and indeed, we have $L^{S}\left(\sigma^{\prime}, s\right)=L^{S}(\sigma, s)$, for a large enough finite set of places $S$, including the archimedean ones. Since $\sigma$ is globally generic, 
it follows, by a theorem of Shahidi [1981], that $L^{S}(\sigma, s)$ does not have a zero at $s=1$. This will prove that $m_{0}(\pi)=0$ for all irreducible, globally generic, cuspidal, automorphic representations $\pi$ of $\mathrm{GSp}_{4}(\mathbb{A})$. We remark that the work in [Kudla et al. 1992] was carried out under the assumption that $k$ is totally real, so we have to make this assumption as well. This is the only place where this restriction is needed.

To finish the proof, we assume that there exists an irreducible cuspidal automorphic representation $\pi^{\prime}$ of $\mathrm{GSp}_{4}(\mathbb{A})$ satisfying Properties (a) and (b), such that $V_{\pi^{\prime}} \subset L_{0}^{2}\left(\mathrm{GSp}_{4}, \xi\right)$. Consider the restriction map from $\mathrm{GSp}_{4}(\mathbb{A})$ to $\mathrm{Sp}_{4}(\mathbb{A})$

$$
\left.f^{\prime} \in V_{\pi^{\prime}} \mapsto f^{\prime}\right|_{\mathrm{Sp}_{4}(\mathbb{A})} .
$$

This is an $\operatorname{Sp}_{4}(\mathbb{A})$-equivariant map. Let $\left[V_{\pi^{\prime}}\right]_{\mathrm{Sp}_{4}(\mathbb{A})}$ be the subspace generated by

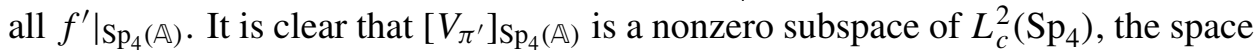
of all cuspidal automorphic functions on $\operatorname{Sp}_{4}(\mathbb{A})$. If we define $L_{0}^{2}\left(\operatorname{Sp}_{4}\right)$ to be the subspace of $L_{c}^{2}\left(\mathrm{Sp}_{4}\right)$ consisting of all cuspidal automorphic functions having (identically) zero Whittaker-Fourier coefficients with respect to any generic character for $\mathrm{Sp}_{4}(\mathbb{A})$, then $\left[V_{\pi^{\prime}}\right] \mathrm{Sp}_{4}(\mathbb{A})$ is included in $L_{0}^{2}\left(\mathrm{Sp}_{4}\right)$. Hence we may decompose

$$
\left[V_{\pi^{\prime}}\right]_{\mathrm{Sp}_{4}(\mathrm{~A})}=\bigoplus_{j} V_{\sigma_{j}^{\prime}},
$$

where $V_{\sigma_{j}^{\prime}}$ are irreducible subspaces of $L_{0}^{2}\left(\mathrm{Sp}_{4}\right)$, corresponding to irreducible cuspidal automorphic representations $\sigma_{j}^{\prime}$ of $\operatorname{Sp}_{4}(\mathbb{A})$. We want to show that the $\sigma_{j}^{\prime}$ satisfy Properties 1, 2, and 3.

It is easy to see that Property 3 holds for $\sigma_{j}^{\prime}$, since $\left[V_{\pi^{\prime}}\right]_{\mathrm{Sp}_{4}(\mathrm{~A})} \subset L_{0}^{2}\left(\mathrm{Sp}_{4}\right)$. Next, we show that the $\sigma_{j}^{\prime}$ satisfy Property 1 , i.e., the $\sigma_{j}^{\prime}$ are locally generic at all places. From (5), it follows that, for each $j$, there is a nontrivial $\mathrm{Sp}_{4}(\mathbb{A})$-homomorphism from $V_{\pi^{\prime}}$ onto $V_{\sigma_{j}^{\prime}}$. This implies that

$$
\operatorname{Hom}_{\mathrm{Sp}_{4}(\mathrm{~A})}\left(V_{\pi^{\prime}}, V_{\sigma_{j}^{\prime}}\right) \neq 0 \text {. }
$$

Let $\mathscr{P}_{j}: V_{\pi^{\prime}} \rightarrow V_{\sigma_{j}^{\prime}}$ be a nonzero projection in (6). Then we may restrict $\mathscr{P}_{j}$ to one place, as follows. Fix $\phi_{v}=\phi_{v, 0} \in V_{\pi_{v}}$, for all $v \neq v_{0}$, such that $\phi_{v}$ is a previously chosen unramified vector, at almost all finite places, and for $\phi_{v_{0}} \in V_{\pi_{v_{0}}}$, we define

$$
\mathscr{P}_{j, v_{0}}\left(\phi_{v_{0}}\right)=\mathscr{P}_{j}\left(\otimes_{v} \phi_{v}\right)
$$

There is a choice of the $\phi_{v, 0}$, for $v \neq v_{0}$, such that $\mathscr{P}_{j, v_{0}}$ is nonzero, and then it defines a nonzero element in

$$
\operatorname{Hom}_{\mathrm{Sp}_{4}\left(k_{v_{0}}\right)}\left(V_{\pi_{v_{0}}^{\prime}}, V_{\sigma_{j}^{\prime}}\right)
$$


As a representation of $\mathrm{Sp}_{4}\left(k_{v_{0}}\right), V_{\sigma_{j}^{\prime}}$ is an isotypic module of $\mathrm{Sp}_{4}\left(k_{v_{0}}\right)$, and is a multiple of $\sigma_{j, v_{0}}^{\prime}$. Hence there is a nonzero element in

$$
\operatorname{Hom}_{\mathrm{Sp}_{4}\left(k_{v_{0}}\right)}\left(V_{\pi_{v_{0}}^{\prime}}, V_{\sigma_{j, v_{0}}^{\prime}}\right) \text {. }
$$

This works for all places $v_{0}$. As a representation of $\operatorname{Sp}_{4}\left(k_{v}\right)$, the restriction of $\pi_{v}^{\prime}$ to $\operatorname{Sp}_{4}\left(k_{v}\right)$ decomposes into a direct sum of irreducible admissible representations of $\mathrm{Sp}_{4}\left(k_{v}\right)$. Clearly each summand is generic with respect to a certain generic character of $U\left(k_{v}\right)$. Hence, at each place $v, \sigma_{j, v}^{\prime}$ is generic with respect to some generic character of $U\left(k_{v}\right)$. This is Property 1 .

It remains to show that the $\sigma_{j}^{\prime}$ satisfy Property 2. Since $\pi^{\prime}$ enjoys Property (a), we know that the irreducible, globally generic, cuspidal, automorphic representation $\pi$ of $\mathrm{GSp}_{4}(\mathbb{A})$ is equivalent to $\pi^{\prime}$. We want to construct an irreducible, cuspidal, automorphic representation $\sigma$ of $\operatorname{Sp}_{4}(\mathbb{A})$, which is globally generic with respect to some generic character, and such that $\sigma$ is nearly equivalent to $\sigma_{j}^{\prime}$.

Consider the restriction map from $\mathrm{GSp}_{4}(\mathbb{A})$ to $\mathrm{Sp}_{4}(\mathbb{A})$ for $\pi$ as before, i.e.,

$$
\left.f \in V_{\pi} \mapsto f\right|_{\operatorname{Sp}_{4}(\mathbb{A})} \in\left[V_{\pi}\right]_{\mathrm{Sp}_{4}(\mathbb{A})} .
$$

There exists $f \in V_{\pi}$ such that $\left.f\right|_{\mathrm{Sp}_{4}(\mathrm{~A})}$ has a nonzero Whittaker-Fourier coefficient on $\mathrm{Sp}_{4}(\mathbb{A})$, with respect to a given generic character of $U(\mathbb{A})$. As before, we have

$$
\left[V_{\pi}\right]_{\mathrm{Sp}_{4}(\mathbb{A})}=\bigoplus_{j} V_{\sigma_{j}}
$$

where $\sigma_{j}$ are irreducible, cuspidal, automorphic, representations of $\operatorname{Sp}_{4}(\mathbb{A})$. This is formula (5) for $V_{\pi}$. Since $\left[V_{\pi}\right]_{\mathrm{Sp}_{4}(\mathrm{~A})}$ has nonzero Whittaker-Fourier coefficients with respect to a certain generic character of $U(\mathbb{A})$, there exists at least one summand in (7) having nonzero Whittaker Fourier coefficients with respect to this character. Such a summand $\sigma$ is an irreducible, cuspidal, automorphic representation of $\mathrm{Sp}_{4}(\mathbb{A})$, which is globally generic with respect to some generic character. We want to show that the $\sigma_{j}^{\prime}$ are nearly equivalent to $\sigma$.

For any irreducible admissible, representation $\Pi$ of $\mathrm{GSp}_{4}(\mathbb{A})$, which may not be automorphic, the restriction of $\Pi$ to $\operatorname{Sp}_{4}(\mathbb{A})$ is a direct sum of irreducible admissible representations of $\operatorname{Sp}_{4}(\mathbb{A})$. At a local unramified place $v$, the restriction of $\Pi_{v}$ to $\mathrm{Sp}_{4}\left(k_{v}\right)$ has one and only one spherical irreducible summand with respect to $\operatorname{Sp}_{4}\left(\mathrm{O}_{v}\right)$. More precisely, for an irreducible unramified representation $\Pi_{v}$ of $\mathrm{GSp}_{4}\left(k_{v}\right)$, consider its restriction of to $\mathrm{Sp}_{4}\left(k_{v}\right)$

$$
\left.\Pi_{v}\right|_{\operatorname{Sp}_{4}\left(k_{v}\right)}=\bigoplus_{j} \sigma_{j, v} .
$$

Exactly one of the summands in (8) is unramified with respect to $\mathrm{Sp}_{4}\left(\mathrm{O}_{v}\right)$. The reason for this is simple, and we thank the referee for pointing it to us. We may 
assume that $\Pi_{v}$ is an irreducible subrepresentation of $\operatorname{Ind}_{B_{v}} \mathrm{GSp}_{4}\left(k_{v}\right) \chi$, where $\chi$ is an unramified character. Consider the map given by restriction of functions to $\mathrm{Sp}_{4}\left(k_{v}\right)$ :

$$
\operatorname{Ind}_{B_{v}}^{\mathrm{GSS}_{4}\left(k_{v}\right)} \chi \longrightarrow \operatorname{Ind}_{B_{v} \cap \mathrm{Sp}_{4}\left(k_{v}\right)}^{\mathrm{Sp}_{4}\left(k_{v}\right)} \chi^{\prime}
$$

where $\chi^{\prime}$ is the restriction of $\chi$ to $B_{v} \cap \mathrm{Sp}_{4}\left(k_{v}\right)$. This map is clearly $\mathrm{Sp}_{4}\left(k_{v}\right)$ equivariant. It is injective since $\mathrm{GSp}_{4}\left(k_{v}\right)=B_{v} \mathrm{Sp}_{4}\left(k_{v}\right)$. Since the right-hand side of (9) has a one-dimensional space of $\mathrm{Sp}_{4}\left(\mathrm{O}_{v}\right)$-fixed vectors, so does the left-hand side, and hence $\Pi_{v}$, as well.

We conclude that the irreducible summands in the restriction of $\Pi$ to $\operatorname{Sp}_{4}(\mathbb{A})$ are nearly equivalent, since they share the same unramified local component at all local unramified places.

Now we apply this to the case of $\pi \cong \pi^{\prime}$. It follows that, as irreducible admissible representations of $\operatorname{Sp}_{4}(\mathbb{A}), \sigma_{j}^{\prime}$ occurs in the restriction of $\pi^{\prime}$ to $\mathrm{Sp}_{4}(\mathbb{A})$ and $\sigma$ occurs in the restriction of $\pi$ to $\operatorname{Sp}_{4}(\mathbb{A})$. Hence $\sigma$ must be nearly equivalent to $\sigma_{j}^{\prime}$. This proves that $\sigma_{j}^{\prime}$ enjoys Property 2. This completes the proof of the Theorem.

We remark that the strong multiplicity-one theorem for globally generic cuspidal representations of $\mathrm{GSp}_{4}(\mathbb{A})$ was proved in [Soudry 1987], namely, two irreducible, automorphic, cuspidal, globally generic representations $\pi_{1}, \pi_{2}$ of $\mathrm{GSp}_{4}(\mathbb{A})$ that are nearly equivalent are isomorphic. From the Theorem in the present paper it also follows that the two representations are, in fact, equal, in the sense that $V_{\pi_{1}}=$ $V_{\pi_{2}}$. (In [Soudry 1987] a reference is made [Piatetski-Shapiro 1979] to derive this equality; it was only later that the author noted that the multiplicity-one theorem proved in that reference applies only to the space $L_{0^{\perp}}^{2}\left(\mathrm{GSp}_{4}, \xi\right)$.) We thus get this corollary:

Corollary. Let $\pi_{1}$ and $\pi_{2}$ be two irreducible, automorphic, cuspidal representations of $\mathrm{GSp}_{4}(\mathbb{A})$, which are nearly equivalent, and act in the spaces of cusp forms $V_{\pi_{1}}$ and $V_{\pi_{2}}$ respectively. Assume that $\pi_{1}$ is globally generic and $\pi_{2}$ is abstractly generic. Then $V_{\pi_{1}}=V_{\pi_{2}}$.

Proof. We first show that $\pi_{2}$ is globally generic. Otherwise, it follows that $V_{\pi_{2}}$ is a subspace of $L_{0}^{2}\left(\mathrm{GSp}_{4}, \xi\right)$, where $\xi$ is the central character of $\pi_{2}$ (which is equal to that of $\pi_{1}$ ). Next, we can find $\sigma$ and $\sigma^{\prime}$ as before - that is, satisfying Properties Property 1-Property 3, with $\pi_{1}$ playing the role of $\pi$ and $\pi_{2}$ that of $\pi^{\prime}$. (In our previous argument, we used the isomorphism between $\pi$ and $\pi^{\prime}$ just to conclude that $\pi^{\prime}$ is abstractly generic, and hence $\sigma^{\prime}$ is also locally generic at all places.) This leads to a contradiction as before. Now that $\pi_{2}$ is also globally generic, we can use [Soudry 1987], to conclude that $\pi_{1}$ and $\pi_{2}$ are isomorphic, and then, by the Theorem, we get $V_{\pi_{1}}=V_{\pi_{2}}$. 


\section{References}

[Kudla et al. 1992] S. S. Kudla, S. Rallis, and D. Soudry, "On the degree 5 -function for Sp(2)", Invent. Math. 107:3 (1992), 483-541. MR 93b:11061 Zbl 0776.11028

[Piatetski-Shapiro 1979] I. I. Piatetski-Shapiro, "Multiplicity one theorems", pp. 209-212 in Automorphic forms, representations and L-functions (Corvallis, OR, 1977), vol. 1, edited by A. Borel and W. Casselman, Proc. Sympos. Pure Math. 33, Amer. Math. Soc., Providence, 1979. MR 81m:22027 Zbl 0423.22017

[Ramakrishnan and Shahidi 2007] D. Ramakrishnan and F. Shahidi, "Siegel modular forms of genus 2 attached to elliptic curves", Math. Res. Lett. (2007). To appear. math/0609468

[Shahidi 1981] F. Shahidi, "On certain L-functions", Amer. J. Math. 103:2 (1981), 297-355. MR 82i:10030 Zbl 0467.12013

[Soudry 1987] D. Soudry, "A uniqueness theorem for representations of GSO(6) and the strong multiplicity one theorem for generic representations of GSp(4)", Israel J. Math. 58:3 (1987), 257287. MR 89f:22025 Zbl 0642.22003

Received August 2, 2006.

DIHUA JIANG

SCHOOL OF MATHEMATICS

UNIVERSITY OF MINNESOTA

206 CHURCH STREET S.E.

MinNEAPOLIS, MN 55455

UNITED STATES

dhjiang@math.umn.edu

www.math.umn.edu/ dhjiang

DAVID SOUDRY

SCHOol of MATHEMatical SCIENCES

TEL AVIV University

TEL AVIV 69978

ISRAEL

soudry@post.tau.ac.il 